DRAFT VERSION NOVEMBER 15, 2018

Preprint typeset using $\mathrm{LT}_{\mathrm{E}} \mathrm{X}$ style emulateapj v. 10/10/03

\title{
THE THERMAL EVOLUTION FOLLOWING A SUPERBURST ON AN ACCRETING NEUTRON STAR
}

\author{
ANDREW CUMMING ${ }^{1}$ AND JARED MACBETH \\ Department of Astronomy and Astrophysics, University of California, Santa Cruz, CA 95064; cumming@ucolick.org \\ Draft version November 15, 2018
}

\begin{abstract}
Superbursts are very energetic Type I X-ray bursts discovered in recent years by long term monitoring of X-ray bursters, and believed to be due to unstable ignition of carbon in the deep ocean of the neutron star. In this Letter, we follow the thermal evolution of the surface layers as they cool following the burst. The resulting light curves agree very well with observations for layer masses in the range $10^{25}-10^{26} \mathrm{~g}$ expected from ignition calculations, and for an energy release $\gtrsim 10^{17} \mathrm{erg}$ per gram during the flash. We show that at late times the cooling flux from the layer decays as a power law $F \propto t^{-4 / 3}$, giving timescales for quenching of normal Type I bursting of weeks, in good agreement with observational limits. We show that simultaneous modelling of superburst lightcurves and quenching times promises to constrain both the thickness of the fuel layer and the energy deposited.
\end{abstract}

Subject headings: accretion, accretion disks-X-rays:bursts-stars:neutron

\section{INTRODUCTION}

Type I X-ray bursts from accreting neutron stars in low mass X-ray binaries involve unstable thermonuclear burning of accreted hydrogen $(\mathrm{H})$ and helium $(\mathrm{He})$ (Lewin, van Paradijs, \& Taam 1995). In the last few years, long term monitoring of X-ray bursters by BeppoSAX and the Rossi X-Ray Timing Explorer (RXTE) has revealed a new class of very energetic Type I X-ray bursts, now known as "superbursts" (see Strohmayer \& Bildsten 2003; Kuulkers 2003 for reviews). The $10^{42}$ erg energies and several hour durations of superbursts are 100-1000 times greater than usual Type I bursts. In addition, they are rare: so far 8 have been seen from 7 sources, with recurrence times not well-constrained, but estimated as $\sim 1$ year (Kuulkers 2002; in 't Zand et al. 2003; Wijnands 2001), instead of hours to days for usual Type I bursts.

The current picture is that superbursts are due to unstable ignition of carbon at densities $\rho \sim 10^{8}-10^{9} \mathrm{~g} \mathrm{~cm}^{-3}$. Hydrogen and helium burn at $\rho \sim 10^{5}-10^{6} \mathrm{~g} \mathrm{~cm}^{-3}$ via the rp-process (Wallace \& Woosley 1981), producing chiefly heavy elements beyond the iron group (including nuclei as massive as $A=104$; Schatz et al. 2001), but with some residual carbon (mass fraction $\left.X_{C} \sim 0.01-0.1\right)$ (Schatz et al. 2003). Cumming \& Bildsten (2001) (hereafter CB01) showed that this small amount of carbon can ignite unstably once the mass of the ash layer reaches $\sim 10^{25} \mathrm{~g}$ (see also Strohmayer \& Brown 2002). This fits well with observed superburst energies for $X_{C} \approx 0.1$ and an energy release from the nuclear burning of $1 \mathrm{MeV}$ per nucleon. The low thermal conductivity of the rp-process ashes gives a large temperature gradient and ignition at the required mass (CB01). The heavy nuclei may also photodisintegrate to iron group during the flash, enhancing the nuclear energy release (Schatz, Bildsten, \& Cumming 2003). Therefore superbursts offer an opportunity to study the rp-process ashes.

Previous authors used one-zone models to estimate the time-dependence of the flash (CB01; Strohmayer \& Brown 2002). In this paper, we present the first multi-zone models of the cooling phase of superbursts. Unlike normal Type I bursts, the time to burn the fuel is much less than the convective turnover time. We therefore assume that the fuel burns locally and instantaneously in place, without significant verti-

\footnotetext{
${ }^{1}$ Hubble Fellow
}

cal mixing. We do not calculate ignition conditions, but rather treat the amount of energy deposited and the thickness of the fuel layer as free parameters ${ }^{2}$. In $\$ 2$, we describe our calculations of the subsequent thermal evolution of the layer, and present a simple analytic model which helps to understand the numerical results. At late times, the flux evolves as a power law in time rather than the exponential decay found by CB01 for a one-zone model. In $\S 3$, we use the long term flux evolution of the layer to predict the timescale of quenching of Type I bursts after the superburst, and compare to observations.

\section{TIME EVOLUTION OF THE SUPERBURST}

After the fuel burns, the cooling of the layer is described by the entropy equation

$$
c_{P} \frac{\partial T}{\partial t}=-\epsilon_{\nu}-\frac{1}{\rho} \frac{\partial F}{\partial r}
$$

where the heat flux is $F=-K(\partial T / \partial r)$, and $\epsilon_{\nu}$ is the neutrino energy loss rate. The layer remains in hydrostatic balance, in which case a useful independent coordinate is the column depth $y$ into the star (units: $\mathrm{g} \mathrm{cm}^{-2}$ ), where $d y=-\rho d r$, giving a pressure $P=g y$. The surface gravity is $g=\left(G M / R^{2}\right)(1+z)$, where $1+z=\left(1-2 G M / R c^{2}\right)^{-1 / 2}$ is the gravitational redshift factor. In this paper, we assume $M=1.4 M_{\odot}$ and $R=10 \mathrm{~km}$, giving $z=0.31$ and $g_{14}=g / 10^{14} \mathrm{~cm} \mathrm{~s}^{-2}=2.45$.

To find the temperature profile just after the fuel burns, we deposit an energy $E_{\text {nuc }}=E_{17} 10^{17} \mathrm{erg} \mathrm{g}^{-1}$ throughout the layer. Since carbon burning to iron gives $\approx 10^{18} \mathrm{erg} \mathrm{g}^{-1}$, we expect $E_{17}=1$ to correspond to $X_{C} \approx 0.05-0.1$, depending on how much energy is contributed by photodisintegration (Schatz et al. 2003). At each depth, we calculate the temperature of the layer, $T_{f}$, from $\int_{T_{i}}^{T_{f}} c_{P} d T=E_{\text {nuc }}$, where $T_{i}$ the initial temperature. Following CB01, a simple analytic estimate of $T_{f}$ is as follows. The electrons are degenerate and relativistic for $\rho \gtrsim 10^{7} \mathrm{~g} \mathrm{~cm}^{-3}$, giving $\rho Y_{e}=1.5 \times 10^{8} \mathrm{~g} \mathrm{~cm}^{-3} P_{26}^{3 / 4}$, Fermi energy $E_{F}=2.7 \mathrm{MeV} P_{26}^{1 / 4}$, and pressure scale height

\footnotetext{
${ }^{2}$ This is a similar approach to Eichler \& Cheng (1989) who studied the thermal response of a neutron star to energy deposition at different depths. However, the transient events they consider are less energetic than superbursts.
} 


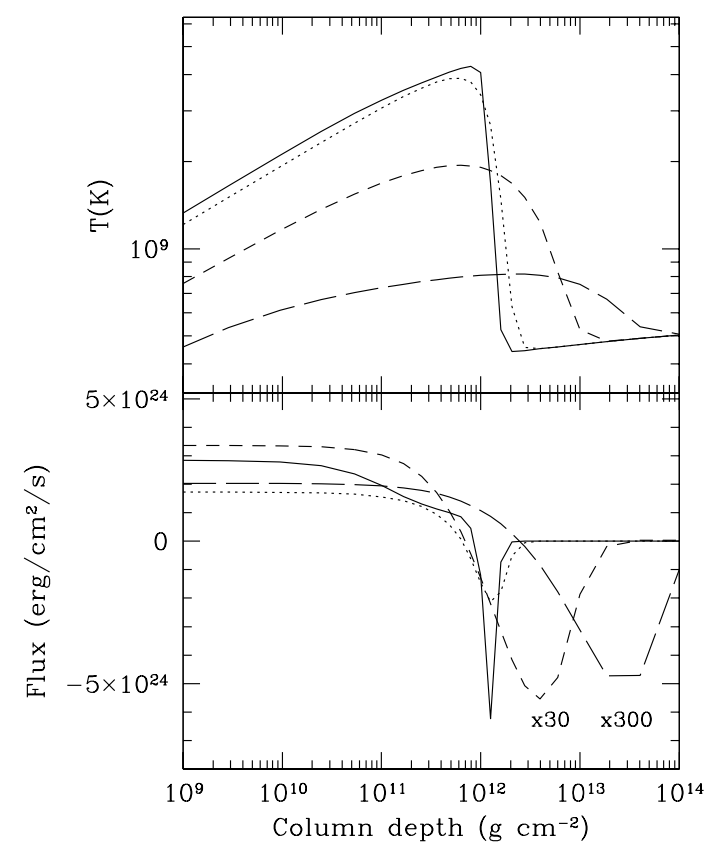

FIG. 1.- Temperature and flux profiles for $y_{b}=10^{12} \mathrm{~g} \mathrm{~cm}^{-2}, E_{17}=1$, and $t=10$ minutes (solid lines), 1 hour (dotted lines), 1 day (short-dashed lines), and 10 days (long-dashed lines) after ignition. In the lower panel, the $t=1$ hour (1 day) flux profile is shown scaled by a factor of 30 (300).

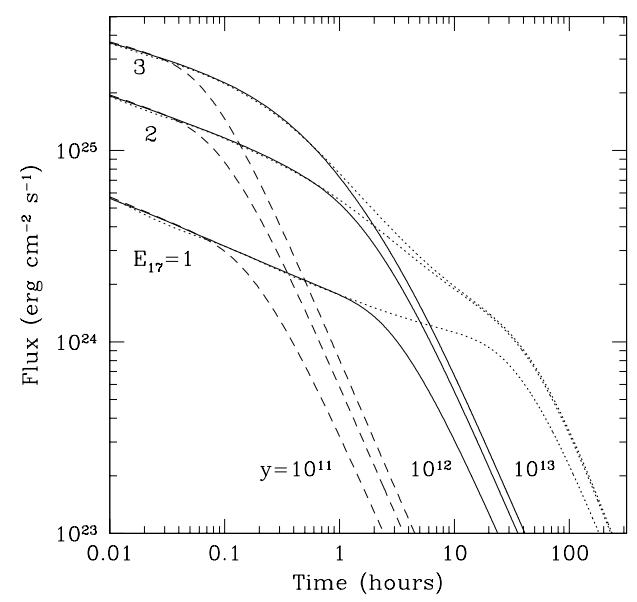

FIG. 2.- Flux from the surface against time for different values for the energy release $E_{17}$ and layer thickness $y_{b}$ (in $\mathrm{g} \mathrm{cm}^{-2}$ ).

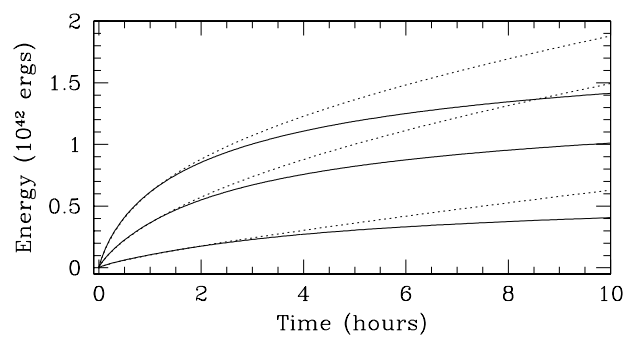

FIG. 3.- Cumulative energy released from the surface as a function of time, for $y_{b}=10^{12}$ (solid lines) and $10^{13} \mathrm{~g} \mathrm{~cm}^{-2}$ (dotted lines) and in each case (bottom to top) $E_{17}=1,2$, and 3 .
$H=y / \rho=67 \mathrm{~m}_{26}^{1 / 4} Y_{e} / g_{14}$, where $P=P_{26} 10^{26} \mathrm{erg} \mathrm{cm}^{-3}$. The heat capacity $c_{P}$ is determined mainly by the electrons, $c_{P} \approx$ $\pi^{2}\left(Y_{e} k_{B} / m_{p}\right)\left(k_{B} T / E_{F}\right)=2.6 \times 10^{7} \mathrm{erg} \mathrm{g}^{-1} \mathrm{~K}^{-1} T_{9} Y_{e} P_{26}^{-1 / 4}$. Integrating and assuming $T_{i} \ll T_{f}$, we find the convectively stable temperature profile $T_{f}=3.6 \times 10^{9} \mathrm{~K} E_{17}^{1 / 2} P_{26}^{1 / 8} Y_{e}^{-1 / 8}$, insensitive to depth and depending mainly on $E_{17}$.

Our thermal evolution code uses the method of lines, in which the right hand side of equation (1) is differenced over a spatial grid, and the resulting set of ordinary differential equations integrated using a stiff integrator. We choose a uniform grid in $\sinh ^{-1}\left(\log y / y_{b}\right)$, which concentrates grid points around the base of the layer $y_{b}$, resolving the initial temperature discontinuity. We place the outer boundary at $y=10^{8} \mathrm{~g} \mathrm{~cm}^{-2}$, and set flux $\propto T^{4}$ there; at the inner boundary, typically $y \approx 10^{14} \mathrm{~g} \mathrm{~cm}^{-2}$, we assume vanishing flux. We assume the layer is heated before the flash by a $10^{21} \mathrm{erg} \mathrm{cm}^{-2} \mathrm{~s}^{-1}$ flux from the crust, and that all the fuel burns to ${ }^{56} \mathrm{Fe}$. Our results are not sensitive to the details of the grid, or boundary conditions (for times longer than the thermal time at the top zone). We calculate the equation of state, opacity, neutrino emissivity, and heat capacity as described by Schatz et al. (2003).

Figure 1 shows temperature and flux profiles 10 minutes, 1 hour, 1 day, and 10 days after ignition for a model with $E_{17}=1$ and $y_{b}=10^{12} \mathrm{~g} \mathrm{~cm}^{-2}$. Figure 2 shows a series of lightcurves for different $y_{b}$ and $E_{17}$. At early times, as the outer parts of the layer thermally adjust, the radiative flux depends mostly on $E_{17}$. At late times, after the cooling wave reaches the base of the layer, the flux depends mostly on $y_{b}$, and falls off as a power law $F \propto t^{-4 / 3}$. Figure 3 shows the cumulative energy release for $y_{b}=10^{12}$ and $10^{13} \mathrm{~g} \mathrm{~cm}^{-2}$ and $E_{17}=1-3$. In the first few hours, the energy released from the surface is $\approx 10^{42} \mathrm{ergs}$, the exact value being mainly sensitive to $E_{17}$, rather than depth. A significant fraction of the heat is initially conducted inwards and released on a longer timescale, as pointed out by Strohmayer \& Brown (2002).

The physical reason for the late-time power law flux decay is that as time evolves, the peak of the temperature profile moves to greater depths where the thermal timescale to the surface is longer ${ }^{3}$ (see Fig. 1). The simplest analytic model is a slab with constant thermal diffusivity $D$, whose temperature is perturbed close to the surface, for example at a depth $x=a$ (where $x=0$ is the surface). For a delta-function perturbation initially, the temperature evolution is given by the Green's function ${ }^{4}$

$$
T(x, t)=\frac{1}{\sqrt{\pi D t}} \sinh \left(\frac{a x}{2 D t}\right) \exp \left(-\frac{x^{2}+a^{2}}{4 D t}\right)
$$

and the surface flux is $F \propto(\partial T / \partial x)_{x=0} \propto t^{-3 / 2} \exp (-\tau / t)$, where $\tau$ is the thermal time at the initial heating depth $\tau=4 a^{2} / D$. For an initial "top hat" temperature profile $T(x<a)=1, T(x>a)=0$, the surface flux is $F \propto$ $(\tau / t)^{1 / 2}[1-\exp (-\tau / t)]$. For $t<\tau$, before the cooling wave reaches the base of the layer, $F \propto t^{-1 / 2}$; for $t>\tau$, the solution

\footnotetext{
${ }^{3}$ A similar problem is ohmic decay of crustal magnetic fields, where power law decay is also expected (Sang \& Chanmugam 1987; Urpin, Chanmugam, \& Sang 1994)

${ }^{4}$ A simple way to obtain this result is to apply the method of images to the Green's function for an unbounded domain $T(x, t) \propto t^{-1 / 2} \exp \left(-x^{2} / 4 D t\right)$. Eichler \& Cheng (1989) derive a similar result for a power law dependence of conductivity on depth, which also shows self-similar behavior at late times (see Lyubarsky, Eichler, \& Thompson 2002 for a recent application to cooling of SGR 1900+14 after an outburst).
} 
is independent of the initial temperature profile, and $F \propto t^{-3 / 2}$.

The numerical results show a similar behavior, although with different power law indices. The relevant timescale in this case is $t_{\text {cool }}=H^{2} / D$, where $D=K / \rho c_{P}$. The electron conductivity is $K=\pi^{2} n_{e} k_{B}^{2} T / 3 m_{\star} \nu_{c}$, where $m_{\star}=E_{F} / c^{2}$, and $\nu_{c}$ is the electron collision frequency. When electrons dominate the heat capacity, the thermal diffusivity takes the particularly simple temperature-independent form $D=c^{2} / 3 \nu_{c}$. For electron-ion collisions, $\nu_{c}=9.3 \times 10^{16} \mathrm{~s}^{-1} P_{26}^{1 / 4}\left\langle Z^{2} / A\right\rangle \Lambda_{e i} / Y_{e}$ (e.g. see Appendix of Schatz et al. 1999), giving

$$
t_{\text {cool }}=3.8 \mathrm{hrs} y_{12}^{3 / 4}\left(\frac{Y_{e}\left\langle Z^{2} / A\right\rangle \Lambda_{e i}}{6}\right)\left(\frac{g_{14}}{2.45}\right)^{-5 / 4}
$$

(see also eq. [10] of CB01), where we insert the appropriate numbers for ${ }^{56} \mathrm{Fe}$ composition. The simple "top hat" solution for constant conductivity motivates a fit to the numerical solutions,

$$
F_{25}=0.2 t_{\mathrm{hr}}^{-0.2} E_{17}^{7 / 4}\left[1-\exp \left(-0.63 t_{\mathrm{cool}}^{4 / 3} E_{17}^{-5 / 4} t_{\mathrm{hr}}^{-1.13}\right)\right],
$$

where $t_{\mathrm{hr}}=t / 1$ hour. For $t>t_{\mathrm{cool}}, F_{25}=0.13 E_{17}^{1 / 2}\left(t / t_{\mathrm{cool}}\right)^{-4 / 3}$. The transition from $F \propto t^{-0.2}$ to $F \propto t^{-4 / 3}$ occurs when $t / t_{\mathrm{cool}} \approx E_{17}^{-1.1}$

Equation (4) fits the numerical results to better than a factor of two for models without substantial neutrino emission. As emphasised by Strohmayer \& Brown (2002), neutrino cooling is important for large carbon fractions: it depresses the flux at $t \approx 5-10$ hours for the models with $E_{17}=2$ and 3, $y=10^{13} \mathrm{~g} \mathrm{~cm}^{-2}$ in Figure 2. Whenever neutrinos dominate the cooling, the peak temperature is large enough that emission is by pair annhilation. A good fit to the neutrino energy loss rate is $\epsilon_{\nu} \approx 10^{4} \mathrm{erg} \mathrm{g}^{-1} \mathrm{~s}^{-1} T_{9}^{12} y_{12}^{-3 / 2}$, giving a cooling time $t_{\nu}=c_{P} T / \epsilon_{\nu}=2.5 \times 10^{12} \mathrm{~s}_{12}^{5 / 4} T_{9}^{-10}$. Inserting the peak temperature from equation (2) gives $t_{\nu} \approx 300 \mathrm{hrs} E_{17}^{-5}$. Neutrinos dominate when $t_{\nu}<t_{\text {cool }}$, or when $E_{17}>2.3 y_{12}^{-3 / 20}$.

\section{COMPARISON TO OBSERVATIONS}

The cooling curves in Figure 2 compare well with observed lightcurves, including a rapid initial decay on hour timescales, followed by an extended tail of emission (as observed following some superbursts, e.g. KS 1731-260, Kuulkers et al. 2002; Ser X-1, Cornelisse et al. 2002). We will present a detailed comparison with the observed superburst lightcurves in a future paper. The initial decay from the peak depends mostly on $E_{17}$, and so it should be possible to constrain the amount of fuel consumed in the superburst. Our models do not resolve the peak itself, since this depends on the details of how the burning propagates out to the surface; however, for $E_{17} \gtrsim 2-3$, the flux exceeds the Eddington flux, $F_{\mathrm{Edd}}=3 \times 10^{25} \mathrm{erg} \mathrm{cm}^{-2} \mathrm{~s}^{-1} /(1+X)$, where $X$ is the $\mathrm{H}$ fraction, for timescales of minutes. Superburst peak luminosities are generally less than the Eddington luminosity (Kuulkers 2003 ), implying $E_{17} \lesssim 2$. The one exception is the superburst from 4U 1820-30, which showed dramatic photospheric radius expansion lasting for several minutes (Strohmayer \& Brown 2002). This is consistent with the proposal that this source, which accretes and burns He rich material, produces large quantities of carbon (Strohmayer \& Brown 2002; Cumming 2003a). The transition to the late-time power law occurs after $t \approx 4 \mathrm{~h} E_{17}^{-1.11} y_{12}^{3 / 4}$ (eq. [5]), which corresponds to $F_{25} \approx 0.13 E_{17}^{2}$. It may therefore be possible to measure the

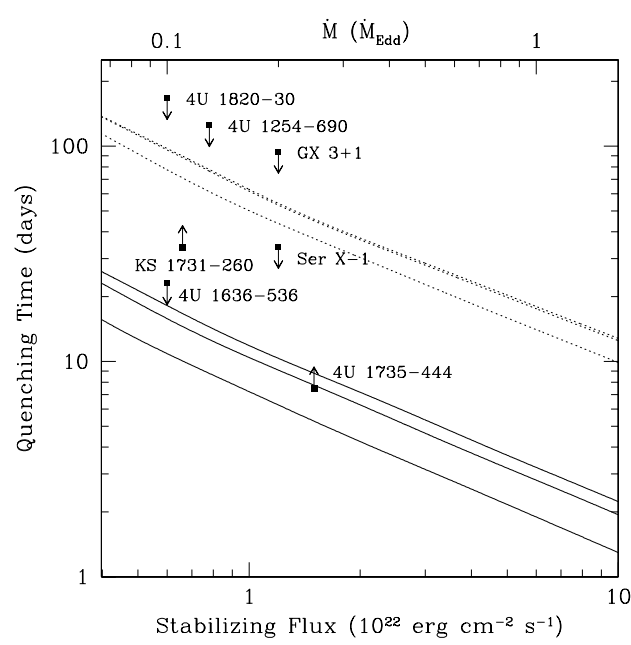

FIG. 4.- Observed and predicted quenching timescales of normal Type I bursts following a superburst. We show the predicted quenching time for $y_{b}=10^{12}$ (solid lines) and $10^{13} \mathrm{~g} \mathrm{~cm}^{-2}$ (dotted lines) as a function of both the critical flux needed to stabilize $\mathrm{H} / \mathrm{He}$ burning $F_{\text {crit }}$, and the accretion rate (given in terms of $F_{\text {crit }}$ by eq. [5]). For each value of $y_{b}$, we show curves for (bottom to top) $E_{17}=1,2$ and 3. The observed upper or lower limits on $\mathrm{t}_{\text {quench }}$, and the estimated $\dot{M}$, are taken from Table 1 of Kuulkers 2003, with an updated value for 4U 1636-53 (Kuulkers, private communication).

power law decay using superburst tails, although this depends upon being able to subtract out the underlying accretion luminosity, $F_{\text {accr }, 25} \approx 0.1\left(\dot{M} / 0.1 \dot{M}_{\text {Edd }}\right)$, in a reliable way.

Another way to probe the late-time cooling is to use the remarkable observation that Type I bursts disappear (are "quenched") for $t_{\text {quench }} \approx$ weeks following the superburst (e.g. Kuulkers 2003). CB01 proposed that the cooling flux from the superburst temporarily stabilizes the $\mathrm{H} / \mathrm{He}$ burning. An estimate of the critical stabilizing flux, $F_{\text {crit }}$, is as follows. The condition for temperature fluctuations to grow, and unstable He ignition to occur, is $\nu \epsilon_{3 \alpha}=\eta \epsilon_{\text {cool }}$ (Fushiki \& Lamb 1987), where $\epsilon_{3 \alpha}$ is the triple alpha ( $\left.3 \alpha\right)$ energy production rate, $\epsilon_{\mathrm{cool}}$ is a local approximation to the cooling rate, and $\nu$ and $\eta$ are the respective temperature sensitivities. For a large flux from below, the He burns stably before reaching this ignition condition, at a depth where the time to accumulate the layer equals the He burning time, $y / \dot{m}=Y Q_{3 \alpha} / \epsilon_{3 \alpha}$, where $Y$ is the He mass fraction, $\dot{m}$ is the local accretion rate per unit area, and $Q_{3 \alpha}=5.84 \times 10^{17} \mathrm{erg} \mathrm{g}^{-1}=0.606 \mathrm{MeV}$ per nucleon is the $3 \alpha$ energy release. At the transition from unstable to stable burning, both criteria are satisfied at the base of the $\mathrm{H} / \mathrm{He}$ layer. Using the first condition to eliminate $\epsilon_{3 \alpha}$ from the second, and writing $\epsilon_{\text {cool }} \approx F / y$, gives $F=\nu \dot{m} Q_{3 \alpha} Y / \eta=$ $6.2 \times 10^{22} \mathrm{erg} \mathrm{cm}^{-2} \mathrm{~s}^{-1}\left(\dot{m} / \dot{m}_{\mathrm{Edd}}\right)(Y / 0.3)(\nu / 4 \eta)$. Some of this flux is provided by hot $\mathrm{CNO}$ burning of accreted $\mathrm{H}, F_{H} \approx$ $\epsilon_{H} y=5.8 \times 10^{21} \mathrm{erg} \mathrm{cm}^{-2} \mathrm{~s}^{-1} y_{8}(Z / 0.01)$ (Cumming \& Bildsten 2000; $Z$ is the metallicity); the remainder is $F_{\text {crit }}=F-F_{H}$. This estimate agrees well with a more detailed calculation using the ignition models of Cumming \& Bildsten (2000), in which we find $F_{\text {crit }} \approx \dot{m} Q_{3 \alpha} \approx 0.7 \mathrm{MeV}$ per accreted nucleon, almost independent of $\dot{M}$. Therefore,

$$
F_{\text {crit }, 22} \approx 6\left(\dot{m} / \dot{m}_{\text {Edd }}\right)
$$

(see also Paczynski 1983a; Bildsten 1995). Equation (5) in 
the limit $t \gg t_{\text {cool }}$ gives

$$
t_{\text {quench }}=38 t_{\text {cool }} F_{\text {crit }, 22}^{-3 / 4} E_{17}^{3 / 8}=6 \text { days } y_{12}^{3 / 4} F_{\text {crit }, 22}^{-3 / 4} E_{17}^{3 / 8},
$$

which gives $t_{\text {quench }}$ in terms of the thickness of the layer and energy release.

Figure 5 compares the predicted and observed quenching times. The observations of $t_{\text {quench }}$ and accretion rates (used to find $F_{\text {crit }}$ from eq. [5]) are taken from Kuulkers (2003) (except for 4U 1636-53, which has a revised upper limit of 23 days, Kuulkers private communication). The observations are upper or lower limits only: nonetheless, the general agreement is very good and supports the quenching picture suggested by CB01. There is much to learn from a careful comparison of superburst lightcurves and the corresponding quenching times, separately constraining both $E_{17}$ and $y_{b}$.

\section{SUMMARY AND CONCLUSIONS}

We have presented the first multi-zone models of the cooling phase of superbursts. The flux decay is not exponential, but power-law (eq. [5]). For $t<t_{\text {cool }}$, where $t_{\text {cool }}$ is the cooling time at the base of the layer, the flux depends mostly on the energy release $E_{17}$, and is insensitive to depth: the inwards travelling cooling wave does not yet know that the layer has a finite thickness. For $t>t_{\text {cool }}$, the flux decays as a power law $F \propto t^{-4 / 3}$, independent of the initial temperature profile. The power law decay at late times gives predicted Type I burst quenching times of weeks (eq. [6]), consistent with observational limits. Future comparisons of both superburst lightcurves and quenching times with observations will constrain both the thickness of the fuel layer and the energy deposited, particularly when combined with models of normal Type I bursts from the same source (Cumming 2003a,b).

There is still much to be done in terms of theory. Perhaps the most important issues are the physics of the rise (which sets the initial condition for our simulations), and production of the fuel. Important clues to the first are the observed precursors to superbursts, which may be normal Type I bursts ignited by the superburst. Recent progress has been made on the second, with indications from both theory (Schatz et al. 2003; Woosley et al. 2003) and observations (in 't Zand et al. 2003) that stable burning may be required to produce enough carbon to power superbursts. A self-consistent model of $\mathrm{H} / \mathrm{He}$ burning, followed by accumulation and ignition of the ashes may require a better understanding of the transition from unstable to stable burning observed in normal Type I bursting (e.g. Cornelisse et al. 2003).

We thank P. Arras, E. Brown, R. Cornelisse, E. Kuulkers, G. Ushomirsky, S. Woosley, and J. in't Zand for useful comments and discussions. AC is supported by NASA through Hubble Fellowship grant HF-01138 awarded by the Space Telescope Science Institute, which is operated by the Association of Universities for Research in Astronomy, Inc., for NASA, under contract NAS 5-26555. JM acknowledges support from DOE grant No. DE-FC02-01ER41176 to the Supernova Science Center/UCSC.

\section{REFERENCES}

Bildsten, L. 1995, ApJ, 438, 852

Brown, E. F., \& Bildsten, L. 1998, ApJ, 496, 915

Cornelisse, R., Kuulkers, E., in't Zand, J. J. M., Verbunt, F., \& Heise, J. 2002,

A\&A, 382, 174

Cornelisse, R. et al. 2003, A\&A, 405, 1033

Cumming, A. 2003a, ApJ, 595, 1077

Cumming, A. 2003b, in "The Restless High-Energy Universe" (Amsterdam,

May 5-8, 2003), ed. E.P.J. van den Heuvel, J.J.M. in 't Zand, and R.A.M.J. Wijers (astro-ph/0309626)

Cumming, A., \& Bildsten, L. 2001, ApJ, 559, L127 (CB01)

Eichler, D., \& Cheng, A. F. 1989, ApJ, 336, 360

Fushiki, I., \& Lamb, D. Q. 1987, ApJ, 323, L55

in't Zand, J. J. M., Kuulkers, E., Verbunt, F., Heise, J., \& Cornelisse, R. 2003, A\&A, 411, L487

Itoh, N., Hayashi, H., Nishikawa, A., \& Kohyama, Y. 1996, ApJS, 102, 411

Kuulkers, E., 2002, A\&A, 383, L5

Kuulkers, E., 2003, in "The Restless High-Energy Universe" (Amsterdam, May 5-8, 2003), ed. E.P.J. van den Heuvel, J.J.M. in 't Zand, and R.A.M.J. Wijers (astro-ph/0310402,

Kuulkers, E. et al. 2002, A\&A, 382, 503

Lewin, W. H. G., van Paradijs, J., \& Taam, R. E. 1995, in X-Ray Binaries, ed. W. H. G. Lewin, J. van Paradijs, \& E. P. J. van den Heuvel (Cambridge: CUP), 175
Lyubarsky, Y., Eichler, D., \& Thompson, C. 2002, ApJ, 580, L69

Paczynski, B., 1983, ApJ, 264, 282

Paczynski, B., 1983, ApJ, 267, 315

Potekhin, A. Y. \& Chabrier, G. 2000, Phys. Rev. E, 62, 8554

Sang, Y. \& Chanmugam, G. 1987, ApJ, 323, L61

Schatz, H., et al. 2001, Phys. Rev. Lett., 86, 3471

Schatz, H., Bildsten, L., \& Cumming, A. 2003, ApJ, 583, L87

Schatz, H., Bildsten, L., Cumming, A., \& Wiescher, M. 1999, ApJ, 524, 1014

Schatz, H., Bildsten, L., Cumming, A., \& Ouellette, M. 2003, Nucl. Phys. A, 718,247

Strohmayer, T. E., \& Bildsten, L. 2003, in Compact Stellar X-Ray Sources, eds. W.H.G. Lewin and M. van der Klis (Cambridge: Cambridge University Press) astro-ph/0301544

Strohmayer, T. E., \& Brown, E. F., 2002, ApJ, 566, 1045

Urpin, V. A., Chanmugam, G., \& Sang, Y. 1994, ApJ, 433, 780

Wallace, R. K. \& Woosley, S. E. 1981, ApJS, 45, 389

Wijnands, R. 2001, ApJ, 554, L59

Woosley, S. E. et al. 2003, ApJS, in press astro-ph/0307425 